(hep-ph/0012046)

KIAS-P00063

YUMS 00-06

\title{
Majorana Neutrino Masses and Neutrino Oscillations
}

\author{
Sin Kyu Kang ${ }^{a, *}$ and C. S. Kim ${ }^{b, c, \dagger}$ \\ a School of Physics, Korea Institute for Advanced Study, Seoul 130-012, Korea \\ ${ }^{b}$ Physics Department and IPAP, Yonsei University, Seoul 120-749, Korea \\ ${ }^{c}$ Department of Physics, University of Wisconsin, Madison, WI 53706, USA
}

\begin{abstract}
We examine some patterns of Majorana neutrino mass matrix which is compatible with the phenomenological lepton flavor mixing matrix and nonobservation of neutrinoless double beta decay. Imposing $\left(M_{\nu}\right)_{e e}=0$ for the Majorana neutrino mass matrix in the leading order, we obtain a relationship between the solar mixing angle and the neutrino masses $m_{1}$ and $m_{2}$. Additional possible texture zeros are assigned to the mass matrix so as for the nonvanishing $\theta_{13}$ to be predictable in terms of neutrino masses. We also show how three neutrino masses can be predicted from the solar mixing angle and the experimental results of $\Delta m_{\text {sol }}^{2}$ and $\Delta m_{\text {atm }}^{2}$ in this framework, and we discuss naturality of the forms of the mass matrix found in our work.
\end{abstract}

PACS number(s):12.15.Ff, 14.60.Pq

Typeset using REVTEX

*skkang@kias.re.kr

†kim@kimcs.yonsei.ac.kr, http://phya.yonsei.ac.kr/ cskim 
Although the lepton flavor mixing matrix can be constructed based on the neutrino oscillation experimental results, the origin of the lepton flavor mixing, the neutrino masses, and their hierarchical patterns are yet to be understood. As an attempt toward the understanding of the neutrino masses and flavor mixing patterns, the mass matrix ansatz has been studied by many authors [1]. In this approach, the flavor mixing elements are not independent parameters but are presented in terms of mass eigenvalues. The special patterns of the flavor mixing and masses can be achieved by imposing some flavor symmetry or taking texture zeros in the mass matrix as much as possible.

As is well known, current data from the atmospheric [2] and solar neutrino experiments [3] provide convincing evidence that neutrinos may have nonzero masses and oscillate, and terrestrial neutrino experiments [4 6] also lead to meaningful constraints on neutrino masses and mixing:

- (a) The atmospheric neutrino experimental data indicates the near maximal mixing between $\nu_{\mu}$ and $\nu_{\tau}, \sin ^{2} 2 \theta_{a t m} \geq 0.8$, with a mass squared difference $\Delta m_{\text {atm }}^{2} \simeq(0.5 \sim$ 6) $\times 10^{-3} \mathrm{eV}^{2}$ at $90 \% \mathrm{CL}$ [2]. The best fit occurs at $\left(\sin ^{2} 2 \theta_{\text {atm }}=1.0, \Delta m_{\text {atm }}^{2}=\right.$ $\left.2.2 \times 10^{-3} \mathrm{eV}^{2}\right)$.

- (b) The solar neutrino anomaly can be explained through matter enhanced neutrino oscillation [7] if $\Delta m_{\text {sol }}^{2} \simeq(0.4 \sim 1) \times 10^{-5} \mathrm{eV}^{2}$ and $\sin ^{2} 2 \theta_{\text {sol }} \simeq(0.1 \sim 1) \times 10^{-2}$ (small angle MSW $(\mathrm{SMA}))$, or $\Delta m_{\text {sol }}^{2} \simeq(1.5 \sim 10) \times 10^{-5} \mathrm{eV}^{2}, \sin ^{2} 2 \theta_{\text {sol }} \geq 0.6$ (large angle MSW (LMA)), $\Delta m_{\text {sol }}^{2} \sim 10^{-7} \mathrm{eV}^{2}, \sin ^{2} 2 \theta_{\text {sol }} \sim 1.0$ (LOW solution) 8 and through long-distance vacuum oscillation (VO) if $\Delta m_{\text {sol }}^{2} \simeq 10^{-10} \mathrm{eV}^{2}, \sin ^{2} 2 \theta_{\text {sol }} \geq 0.7$.

- (c) Moreover, the CHOOZ experimental results can constrain $\nu_{e}-\nu_{x}$ oscillation with $\Delta m_{\text {atm }}^{2} \geq 10^{-3} \mathrm{eV}^{2}$ [5] but gives no limit for $\Delta m_{\text {atm }}^{2}<10^{-3} \mathrm{eV}^{2}$, and the recent Palo Verde reactor experiment also indicates no atmospheric $\nu_{e}-\nu_{x}$ oscillation for $\Delta m^{2} \geq 1.12 \times 10^{-3}$ and for $\sin ^{2} 2 \theta \geq 0.21$ (for large $\Delta m^{2}$ ) [6]. From those reactor experiments, we can obtain a constraint on the magnitude of $U_{e 3}$, which is turned out to be small, i.e., $\left|U_{e 3}\right| \leq 0.22$. 
- (d) In the case of the LSND experiment at LANL [由], a evidence for $\overline{\nu_{\mu}} \rightarrow \overline{\nu_{e}}$ oscillation has been reported. However, since the LSND result has not yet been independently confirmed by other similar experiments, we do not include it in our analysis.

- (e) Now we assume that there are only three active neutrinos with Majorana masses. For convenience, let us adopt the following convention. The heaviest neutrino mass eigenstate responsible for the atmospheric neutrino anomaly is taken to be $\nu_{3}$, whereas those responsible for the solar neutrino problem are $\nu_{1}$ and $\nu_{2}$. Then, the mass squared differences between two atmospheric neutrinos and two solar neutrinos become $\Delta m_{\text {atm }}^{2} \simeq \Delta m_{32}^{2} \simeq \Delta m_{31}^{2}$ and $\Delta m_{\text {sol }}^{2} \simeq \Delta m_{21}^{2}$, respectively.

Using the above experimental constraints on the neutrino masses and mixing angles, one can construct the phenomenological lepton flavor mixing matrix, as follows:

- (i) Since the best fitted value of the Super-Kamiokande data for the atmospheric neutrino mixing angle corresponds to the maximal mixing, we take $\theta_{23}=\pi / 4$. However, since there are two possibilities for the solar neutrino mixing angle $\theta_{12}$ as shown in the above, we present corresponding elements of the mixing matrix in terms of $\theta_{12} \equiv \theta$.

- (ii) As for the mixing angle $\theta_{13}$ which is related to $U_{e 3}$, there is only upper bound on its value as presented above. As shown in Ref. [9], the fitted values for the oscillation amplitude for solar neutrinos are not greatly affected by the particular value of $s_{13}$ in this case, thus we take $\sin \theta_{13} \equiv \epsilon$ and $\cos \theta_{13} \sim 1$ in the leading order.

Then in general the lepton mixing matrix in the standard parametrization has the form in the leading order 10,

$$
\begin{aligned}
U & =\left(\begin{array}{ccc}
c_{13} c_{12} & c_{13} s_{12} & s_{13} \\
-c_{23} s_{12}-s_{13} s_{23} c_{12} & c_{23} c_{12}-s_{13} s_{23} s_{12} & c_{13} s_{23} \\
s_{23} s_{12}-s_{13} c_{23} c_{12} & -s_{23} c_{12}-s_{13} c_{23} s_{12} & c_{13} c_{23}
\end{array}\right) \\
& =\left(\begin{array}{ccc}
c & s & \epsilon \\
-\frac{1}{\sqrt{2}}(s+c \epsilon) & \frac{1}{\sqrt{2}}(c-s \epsilon) & \frac{1}{\sqrt{2}} \\
\frac{1}{\sqrt{2}}(s-c \epsilon) & -\frac{1}{\sqrt{2}}(c+s \epsilon) & \frac{1}{\sqrt{2}}
\end{array}\right),
\end{aligned}
$$


where $c=\cos \theta_{12}, s=\sin \theta_{12}$, and the neutrino flavor basis is $\left(\nu_{e}, \nu_{\mu}, \nu_{\tau}\right)$. Here, we assume that there is no $\mathrm{CP}$ violation in the lepton sector.

In this Letter, we will examine some patterns of Majorana neutrino mass matrix which is compatible with the above lepton mixing matrix and reflects the predictable framework of neutrino masses. Recently, there has been much work suggesting various textures of neutrino masses by using some phenomenological ansatz [11] or some symmetry arguments [12], such as $S O(10), S O(3), U(2), U(1)$, etc. Here we will take the approach to require texture zeros from the appropriate experimental observations, instead of imposing specific flavor symmetry in the neutrino sector. The general form of mass matrix presented in terms of three neutrino mass eigenvalues will be provided with the help of the lepton mixing matrix. Motivated by non-observation of neutrinoless double beta decay, we impose $\left(M_{\nu}\right)_{e e}=0$ for the Majorana neutrino mass matrix in the leading order, which in turn makes the solar mixing angle simply related to the ratio $m_{1} / m_{2}$. This is consistent as long as $\epsilon^{2}<<m_{2} \sin ^{2} \theta_{12} / m_{3}$. Additional possible texture zeros will be assigned to the mass matrix so as for the mixing parameter $\epsilon$ to be predictable in terms of neutrino mass eigenvalues. We will also show that three neutrino mass eigenvalues can be calculated from the relation for the solar mixing angle and the experimental results of $\Delta m_{\text {sol }}^{2}$ and $\Delta m_{\text {atm }}^{2}$.

A strong constraint on some element of Majorana neutrino mass matrix can come from the experimental results of neutrinoless double beta decay, whose non-observation might serve as a texture zero for the leading order mass matrix. If massive neutrinos are Majorana particles, the matrix element of the neutrinoless double beta decay is proportional to the effective Majorana mass

$$
\left|<m_{\nu}>\right|=\left|\sum_{i} m_{i} U_{e i}^{2}\right|
$$

where $U$ is the lepton mixing matrix that connects the flavor neutrino eigenstates $\nu_{\alpha L}(\alpha=$ $e, \mu, \tau)$ to the mass eigenstates $\nu_{i}(i=1,2,3)$ through the relation

$$
\nu_{\alpha L}=\sum_{i} U_{\alpha i} \nu_{i L}
$$


This effective Majorana mass is equal to the absolute value of the element $\left(M_{\nu}\right)_{e e}$ of the mass matrix in the charged lepton flavor basis, i.e. the mass matrix for the charged leptons in its diagonal basis. The current experimental upper bound on $\left|<m_{\nu}\right\rangle \mid$ is given by [13],

$$
\left|<m_{\nu}>\right| \leq 0.2 \mathrm{eV} \quad(90 \% \text { C.L. }) \text {. }
$$

The GENIUS experiment is expected to be sensitive to $\left|\left\langle m_{\nu}\right\rangle\right|$ as low as 0.01 eV or even $0.001 \mathrm{eV}$ 14.15]. Thus, the magnitude of the element $\left(M_{\nu}\right)_{e e}$ might be strongly constrained by the experimental results of neutrinoless double beta decay. Although it is not yet proved, it is possible to enforce $\left(M_{\nu}\right)_{e e}=0$ for some special pattern of neutrino mixing [16]. In this paper, we require $\left(M_{\nu}\right)_{e e}=0$ in the leading order, from which the solar mixing angle is simply related to the neutrino mass ratio $m_{1} / m_{2}$. We note that although $\left(M_{\nu}\right)_{e e}=0$ in the leading order, there is nonvanishing very small next leading contribution to $\left(M_{\nu}\right)_{e e}$ due to nonzero $\epsilon$ parameter whose magnitude is proportional to $\epsilon^{2}$.

The Majorana neutrino mass matrix in the charged lepton flavor basis can be given by $M_{\mu}=U \cdot D \cdot U^{T}$. The diagonal matrix can be written as $\operatorname{diag}\left[m_{1} e^{i \alpha}, m_{2} e^{i \beta}, m_{3}\right]$, where $m_{i}(i=1,2,3)$ is positive definite. For CP conserving case, the phases $\alpha$ and $\beta$ are taken to be either $\pi$ or 0 . Thus, we can consider the possible three cases:

- Case (1) $M_{\nu}=U \cdot \operatorname{diag}\left[-m_{1}, m_{2}, m_{3}\right] \cdot U^{T}$,

- Case (2) $M_{\nu}=U \cdot \operatorname{diag}\left[m_{1},-m_{2}, m_{3}\right] \cdot U^{T}$,

- Case (3) $M_{\nu}=U \cdot \operatorname{diag}\left[ \pm m_{1}, \pm m_{2}, m_{3}\right] \cdot U^{T}$.

Case (1): Keeping the $\epsilon^{2}$ order, the neutrino mass matrix is presented by

$$
\begin{aligned}
M_{\nu}= & \left(\begin{array}{ccc}
w_{1} & \frac{1}{\sqrt{2}}\left[\epsilon\left(m_{3}-w_{1}\right)+m_{+} c s\right] & \frac{1}{\sqrt{2}}\left[\epsilon\left(m_{3}-w_{1}\right)-m_{+} c s\right] \\
\frac{1}{\sqrt{2}}\left[\epsilon\left(m_{3}-w_{1}\right)+m_{+} c s\right] & \frac{1}{2}\left(m_{3}+w_{2}-2 m_{+} c s \epsilon\right) & \frac{1}{2}\left(m_{3}-w_{2}\right) \\
\frac{1}{\sqrt{2}}\left[\epsilon\left(m_{3}-w_{1}\right)-m_{+} c s\right] & \frac{1}{2}\left(m_{3}-w_{2}\right) & \frac{1}{2}\left(m_{3}+w_{2}+2 m_{+} c s \epsilon\right)
\end{array}\right) \\
& +\epsilon^{2}\left(\begin{array}{ccc}
m_{3}-w_{1} & -\frac{c s}{2 \sqrt{2}} m_{+} & \frac{c s}{2 \sqrt{2}} m_{+} \\
-\frac{c s}{2 \sqrt{2}} m_{+} & -\frac{1}{2}\left(m_{3}-w_{1}\right) & -\frac{1}{2}\left(m_{3}-w_{1}\right) \\
\frac{c s}{2 \sqrt{2}} m_{+} & -\frac{1}{2}\left(m_{3}-w_{1}\right) & -\frac{1}{2}\left(m_{3}-w_{1}\right)
\end{array}\right)
\end{aligned}
$$


where $w_{1}=-m_{1} c^{2}+m_{2} s^{2}, w_{2}=-m_{1} s^{2}+m_{2} c^{2}$ and $m_{+}=m_{1}+m_{2}$. From the condition $\left(M_{\nu}\right)_{e e}=0$ in the leading order, the following relation comes out

$$
\tan \theta_{12}=\tan \theta_{\text {sol }}=\sqrt{\frac{m_{1}}{m_{2}}} .
$$

Thus, in this ansatz, the solar neutrino mixing pattern is attributed to the ratio $m_{1} / m_{2}$. For the hierarchy $m_{1}, m_{2}<<m_{3}$, the natural choice for two additional texture zeros of the mass matrix in the leading order would be $\left[\left(M_{\nu}\right)_{e \tau},\left(M_{\nu}\right)_{\tau e}\right]$ elements, which lead to the following relation;

$$
\epsilon=\frac{\sqrt{m_{1} m_{2}}}{m_{3}}
$$

From the $\mathrm{CHOOZ}$ experimental results, $U_{e 3}$ can be constrained and we obtain

$$
\left|U_{e 3}\right|=\epsilon=\frac{\sqrt{m_{1} m_{2}}}{m_{3}}<0.22 \text {. }
$$

Then, we are led to the leading order neutrino mass matrix in the charged lepton basis presented in terms of the three neutrino mass eigenvalues

$$
M_{\nu}=m_{3}\left(\begin{array}{ccc}
0 & \sqrt{2} \epsilon & 0 \\
\sqrt{2} \epsilon & \frac{1}{2}\left(1+\frac{m_{2}-m_{1}}{m_{3}}\right) & \frac{1}{2}\left(1-\frac{m_{2}-m_{1}}{m_{3}}\right) \\
0 & \frac{1}{2}\left(1-\frac{m_{2}-m_{1}}{m_{3}}\right) & \frac{1}{2}\left(1+\frac{m_{2}-m_{1}}{m_{3}}\right)
\end{array}\right)+\mathcal{O}\left(\epsilon^{2}\right) .
$$

We note that this form of mass matrix is similar pattern of the neutrino mass matrix presented in [17]. From Eq. (8), we see that only the hierarchy $m_{1}, m_{2}<<m_{3}$ is relevant for this form of mass matrix to be consistent with the experimental results. In this ansatz, the maximal mixing of solar neutrino oscillation is attributed to almost degenerate $\nu_{1}$ and $\nu_{2}$, while the small mixing is achieved by the hierarchy of $m_{1}$ and $m_{2}$. For the inverted hierarchy, $m_{1} \sim m_{2}>>m_{3}$, one can have another form of mass matrix with three texture zeros by taking $m_{3} \simeq m_{2}-m_{1}$ :

$$
\begin{aligned}
M_{\nu} \simeq & \left(\begin{array}{ccc}
0 & \frac{1}{\sqrt{2}}\left(\epsilon m_{3}+\sqrt{m_{1} m_{2}}\right) & \frac{1}{\sqrt{2}}\left(\epsilon m_{3}-\sqrt{m_{1} m_{2}}\right) \\
\frac{1}{\sqrt{2}}\left(\epsilon m_{3}+\sqrt{m_{1} m_{2}}\right) & m_{3}-\epsilon \sqrt{m_{1} m_{2}} & 0 \\
\frac{1}{\sqrt{2}}\left(\epsilon m_{3}-\sqrt{m_{1} m_{2}}\right) & 0 & m_{3}-\epsilon \sqrt{m_{1} m_{2}}
\end{array}\right) \\
& +\mathcal{O}\left(\epsilon^{2}\right) .
\end{aligned}
$$


However, in this case, the value of $\epsilon$ is not predicted. If the magnitude of $\epsilon$ is taken to be negligibly small, the form of mass matrix indicates nearly pseudo-Dirac neutrinos.

Case (2): Keeping only to $\mathcal{O}(\epsilon)$, we have

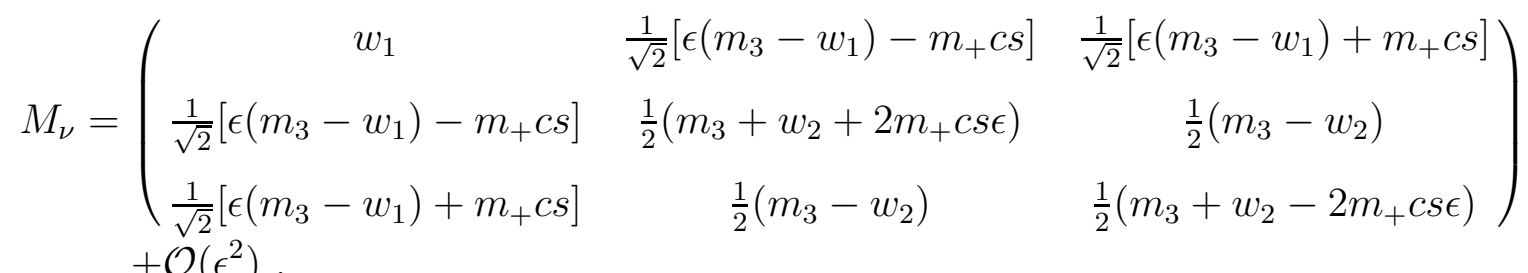

where $w_{1}=m_{1} c^{2}-m_{2} s^{2}, w_{2}=m_{1} s^{2}-m_{2} c^{2}$ and $m_{+}=m_{1}+m_{2}$. The next leading order contribution $O\left(\epsilon^{2}\right)$ is similar to the Case (1). We are also led to the relation Eq. (6) from the condition of no neutrinoless double beta decay in the leading order. The natural choice for two additional texture zeros in this case is to take $\left(M_{e \mu}, M_{\mu e}\right)$ elements to be zero which lead to the same relation as Eq. (7). Then, this form of mass matrix become triangular type of mass matrix:

$$
M_{\nu}=m_{3}\left(\begin{array}{ccc}
0 & 0 & \sqrt{2} \epsilon \\
0 & \frac{1}{2}\left(1-\frac{m_{2}-m_{1}}{m_{3}}\right) & \frac{1}{2}\left(1+\frac{m_{2}-m_{1}}{m_{3}}\right) \\
\sqrt{2} \epsilon & \frac{1}{2}\left(1+\frac{m_{2}-m_{1}}{m_{3}}\right) & \frac{1}{2}\left(1-\frac{m_{2}-m_{1}}{m_{3}}\right)
\end{array}\right)+O\left(\epsilon^{2}\right) .
$$

As one can see, the Case (3) leads to the nonzero effective Majorana mass in the leading order. This is incompatible with our ansatz of no neutrinoless double beta decay in the leading order. Thus, we do not consider this case any more.

Now, let us demonstrate how neutrino masses can be determined from the above results. The numerical values of the mass squared differences $\Delta m_{21}^{2}$ and $\Delta m_{32}^{2}$ can be obtained from the experimental results of $\Delta m_{\text {sol }}^{2}$ and $\Delta m_{\text {atm }}^{2}$, respectively. Since the mixing angle $\sin ^{2} 2 \theta_{\text {sol }}$ is related to the mass eigenvalues $m_{1}$ and $m_{2}$ through the relation (6), combining this with the numerical value of $\Delta m_{21}^{2}$, one can first determine the numerical values of $m_{1}$ and $m_{2}$. Then, the mass eigenvalue $m_{3}$ is determined from $\Delta m_{a t m}^{2}=\Delta m_{32}^{2}$. In this way, one can get possible ranges of three neutrino masses. However, since there are two possibilities for the mixing angle $\theta_{12}$ corresponding to two types of the solar neutrino mixing, we have to 
consider two cases.

(A) Small mixing angle solution: From $\sin ^{2} 2 \theta_{\text {sol }} \simeq 10^{-2}$, we obtain the mass ratio $m_{1} / m_{2} \simeq 0.01$ which implies that the mass hierarchy $m_{1}<<m_{2}$ is required. Combining this with the experimental results for $\Delta m_{\text {sol }}^{2}$ and $\Delta m_{a t m}^{2}$, we obtain

$$
\left(m_{1}, m_{2}, m_{3}\right)=\left[(0.5 \sim 8) \times 10^{-6}, \quad(2 \sim 3) \times 10^{-3}, \quad 0.05\right] \quad \mathrm{eV}
$$

From these results, we can obtain $\epsilon=(0.7 \sim 3) \times 10^{-3}$, which is consistent with the CHOOZ and Palo Verde experimental results.

(B) Large mixing angle solutions: For large mixing of the solar neutrinos, Eq. (6) leads to $m_{1} \simeq m_{2}$. We note that the exact maximal mixing for the solar neutrino oscillation is not realistic for this approach. Taking some value in the allowed region of $\sin ^{2} 2 \theta_{\text {sol }}$ except 1, one can determine three neutrino mass eigenvalues in the same way as the SMA case. For example, if

$$
\left(\sin ^{2} 2 \theta_{\text {sol }}, \quad \Delta m_{\text {sol }}^{2}, \quad \delta m_{\text {atm }}^{2}\right)
$$

are taken to be

$$
\left(0.9, \quad 10^{-4}\left(10^{-10}\right) \mathrm{eV}^{2}, \quad 0.0022 \mathrm{eV}^{2}\right)
$$

respectively for LMA (VO), the allowed neutrino masses are then give by

$$
\left(m_{1}, m_{2}, m_{3}\right)=\left(\begin{array}{llll}
6 \times 10^{-3} & 1 \times 10^{-2} & 0.05 & (\mathrm{LAM}) \\
6 \times 10^{-6} & 1 \times 10^{-5} & 0.05 & (\mathrm{VO})
\end{array}\right) \mathrm{eV}
$$

The prediction of $\epsilon$ is 0.17 and $2 \times 10^{-4}$ for LMA and VO, respectively. Those results are also consistent with the experimental bounds.

Based on the above numerical results, we can estimate the possible effective Majorana mass arising in the next leading order. From Eq. (5), it is given by $\epsilon^{2} m_{3}$ and numerically $(0.2 \sim 5) \times 10^{-7}$ for SMA and $1.5 \times 10^{-3}\left(2 \times 10^{-9}\right)$ for LMA (VO) with $\sin ^{2} 2 \theta_{\text {sol }}=0.9$ Those values are far below the current experimental bound given by Eq. (4). In particular, a new Heidelberg experimental proposal (GENIUS) will allow to increase the sensitivity for 
Majorana neutrino masses from the present level of $0.1 \mathrm{eV}$ down to 0.01 or even $0.001 \mathrm{eV}$. In an extended experiment using 10 tons of ${ }^{76} \mathrm{Ge}$, a sensitivity of $0.001 \mathrm{eV}$ could be reached [15]. Thus, we expect that the test of the prediction of the possible effective Majorana mass for LMA would be accessible in near future.

Now, let us take into account the limit of $\epsilon=0$. Requiring no neutrinoless double beta decay, the mass matrix takes the form for the mass eigenvalues $\left(-m_{1}, m_{2}, m_{3}\right)$ :

$$
M_{\nu}=\left(\begin{array}{ccc}
0 & -\sqrt{\frac{m_{1} m_{2}}{2}} & \sqrt{\frac{m_{1} m_{2}}{2}} \\
-\sqrt{\frac{m_{1} m_{2}}{2}} & \frac{1}{2}\left(m_{3}+m_{1}-m_{2}\right) & \frac{1}{2}\left(m_{3}-m_{1}+m_{2}\right) \\
\sqrt{\frac{m_{1} m_{2}}{2}} & \frac{1}{2}\left(m_{3}-m_{1}+m_{2}\right) & \frac{1}{2}\left(m_{3}+m_{1}-m_{2}\right)
\end{array}\right) .
$$

Since the solar mixing angle is given in terms of $m_{1}$ and $m_{2}$ by Eq. (6), the maximal mixing of solar neutrinos implies $m_{1}=m_{2}$. However, it is not easy to naturally generate $\Delta m_{\text {sol }}^{2}$. For almost bimaximal mixing case [18] which is due to nearly maximal solar mixing, similar to $\epsilon \neq 0$ case, neutrino mass spectrum is predicted providing $\sin ^{2} 2 \theta_{\text {sol }}$ is fixed so that the tiny mass splitting between $m_{1}$ and $m_{2}$ is naturally come out.

At this stage, we address whether the above types of neutrino mass matrices can be obtained from some natural models of lepton masses and mixings. We will, first of all, show that the forms of the mass matrix given by Eqs. $(9,12)$ can naturally be generated from some class of GUT models through a seesaw mechanism [19. As shown in Refs. 19,20, the relevant form of the Dirac neutrino mass matrix is given in a parallel way with the up-type quark mass matrix in the GUT framework,

$$
m_{U} \simeq m\left(\begin{array}{ccc}
0 & 0 & r \\
0 & r & 0 \\
r & 0 & 1
\end{array}\right)
$$

where $r$ is a small parameter of order $\sim(1 / 100-1 / 300) \sim m_{c} / m_{t}$ and the scale $m \sim m_{t} / 3$ where the factor 3 represents the effect of renormalization group equation. Then, the forms of the mass matrix given by Eqs. $(9,12)$ can be obtained through the seesaw relation, $M_{\nu}=m_{U}^{T} M_{R}^{-1} m_{U}$, by taking the following forms of the right-handed majorana mass matrix: 


$$
M_{R} \simeq\left(\begin{array}{ccc}
a M^{\prime} & b M^{\prime} & 0 \\
b M^{\prime} & c M^{\prime} & 0 \\
0 & 0 & M
\end{array}\right), \quad M_{R} \simeq\left(\begin{array}{ccc}
M^{\prime} & 0 & a M \\
0 & 0 & b M \\
a M & b M & c M
\end{array}\right)
$$

where the former corresponds to Eq. (9) and the latter to Eq. (12). We note that the nondiagonal form of the charged lepton mass matrix diagonalized by a mixing matrix with very small off-diagonal elements does not hurt the form of $M_{\nu}$. In order for the GUT scenario to be consistent with the observed bottom-tau mass ratio, it is required that the right-handed Majorana mass of the third generation must be heavier than at least $10^{13} \mathrm{GeV}$ [19,21]. Using the previous numerical results for the three neutrino masses, one can estimate the ranges of the parameters $(a, b, c)$ and $\left(M, M^{\prime}\right)$ and then check whether the scales $M$ and $M^{\prime}$ are compatible with the GUT scenario. While the consistent case for the former happens at the vacuum angle solution whics gives $M \sim 10^{10} \mathrm{GeV}, M^{\prime} \sim 10^{14} \mathrm{GeV}$, the consistent cases for the latter happen at the small mixing and large mixing angle MSW solutions which provide $M \sim 10^{10-11}, M^{\prime} \sim 10^{15-16} \mathrm{GeV}$. Here, the magnitude of $a, b, c$ are determined to be of order $\mathcal{O}(1)$, and the scale $M^{\prime}$ is given by the order of around GUT scale. Since an intermediate scale $10^{10-11} \mathrm{GeV}$ is also naturally viable in the GUT scenario, we may say that the above solutions we found are quite natural. On the other hand, while the form given by Eq. (10) can not be obtained in such a natural way through the canonical seesaw mechanism, it can, as shown in Ref. [22], be achieved in the type II seesaw model with approximate $L_{e}-L_{\mu}-L_{\tau}$.

To justify above ansatz that leads to the proposed form of neutrino mass matrix with three texture zeros and neutrino spectrum, the precise determination of $U_{e 3}$ element as well as the precise experiment for neutrinoless double beta decay will may be essential, which requires several oscillation channels to be probed at the same time. From the fact that the $\nu_{\mu} \rightarrow \nu_{\tau}$ disappearance channel is sensitive only to $\left|U_{\mu 3}\right|^{2}$ and the $\nu_{\mu} \rightarrow \nu_{e}$ appearance channel is sensitive to the product $\left|U_{\mu 3}\right|^{2} \cdot\left|U_{e 3}\right|^{2}$, one can determine $\left|U_{e 3}\right|$ by combining the regions to be probed in both channels. K2K [23] will be expected to perform this, but it does not, at present, sensitivity in the $\nu_{\mu} \rightarrow \nu_{e}$ appearance channel to probe the region of 
$\left|U_{e 3}\right|^{2}$ allowed by Super-Kamiokande, CHOOZ, and Palo Verde [24].

In summary, we have examined some patterns of Majorana neutrino mass matrix which is compatible with the phenomenological lepton flavor mixing matrix and non-observation of neutrinoless double beta decay. We constructed the lepton mixing matrix by taking $\theta_{23}=$ $\pi / 4$ which corresponds to the maximal mixing of the atmospheric neutrinos, $\theta_{12}=\theta_{\text {sol }}$, and allowing non-vanishing very small mixing angle $\theta_{13}$. Imposing $\left(M_{\nu}\right)_{e e}=0$ for the Majorana neutrino mass matrix in the leading order, a relationship between the solar mixing angle and the ratio of the first two neutrino mass eigenvalues $m_{1} / m_{2}$ has been obtained. Additional possible texture zeros have been assigned to the mass matrix so as for the nonvanishing $\theta_{13}$ to be predictable in terms of neutrino masses. We have showed how three neutrino mass eigenvalues can be estimated from the relation for the solar mixing angle and the experimental results of $\Delta m_{\text {sol }}^{2}$ and $\Delta m_{\text {atm }}^{2}$ in this framework. We have also discussed how some forms of the mass matrix found in this paper can be achieved in any natural model of lepton masses.

\section{ACKNOWLEDGMENTS}

We thank V. Barger and K. Whisnant for careful reading of the manuscript and their valuable comments. The work of C.S.K. was supported in part by BK21 Project, in part by Grant No. 2000-1-11100-003-1 and SRC Program of the KOSEF, and in part by the KRF Grants (Project No. 1997-011-D00015 and Project No. 2000-015-DP0077).

Note added: while this paper is being completed, we heard news of recent analysis for solar neutrino oscillation by SuperKamiokande which indicates that LMA is favored at 95\%CL, whereas SMA and VO are disfavored. If it will be conformed in the future, only the part of LMA in our work is relevant. 


\section{REFERENCES}

[1] For reviews, see G. Altarelli and F. Feruglio, Phys. Rep. 320, 295 (1999); H. Fritzsch and Z. Xing, hep-ph/9912358.

[2] Y. Fukuda, et.al., [Super-Kamiokande Collaboration], Phys. Rev. Lett. 81, 1562 (1998).

[3] B. Cleveland et al., Astrophys. J. 496, 505 (1998); K. Hirata et al., [Kamiokande Collaboration], Phys. Rev. Lett. 77, 1683 (1996); W. Hampel et al., [GALLEX Collaboration], Phys. Lett. B447, 127 (1999); J. Abdurashitov et al., [SAGE Collaboration], astro-ph/9907113; Y. Fukuda, et al., [Super-Kamiokande Collaboration], Phys. Rev. Lett. 82, 2430 (1999).

[4] C. Athanassopoulos et al., Phys. Rev. Lett. 81, 1744 (1998).

[5] M. Apollonio et al., [CHOOZ Collaboration], Phys. Lett. B420, 397 (1998); hepex/9907037.

[6] F. Boehm et al., [Palo Verde Collaboration], hep-ex/9912050; hep-ex/0003022.

[7] L. Wolfenstein, Phys. Rev. D 17, 2369 (1978); S. Mikheyev and A. Yu. Smirnov, Yad. Fiz. 42, 1441 (1985); Nuovo Cimento 9 C, 17 (1986).

[8] For recent analyses, see J. N. Bahcall, S. P. Krastev and A. Yu. Smirnov, Phys. Rev. D58, 096016 (1998); M.C. Gonzalez-Garcia et al., Nucl. Phys. B573, 3 (2000); G.L. Fogli et al., Phys. Rev. D62, 013002 (2000).

[9] V. Barger and K. Whisnant, Phys. Rev. D59, 093007 (1999); R. Barbieri et al., JHEP 9812:017, 1998.

[10] See also E. Kh. Akhmedov, Phys. Lett. B467, 95 (1999).

[11] R. Barbieri et al., Phys. Lett. B445, 407 (1999); L. Hall and D. Smith, Phys. Rev. D59, 113013 (1999); E. Malkawi, Phys. Rev. D61, 013006 (1999); K. Kang et al., Phys. Lett. B442, 249 (1998); M. Randhawa, Phys. Rev. D60, 051301 (1999); B. Desai et 
al., hep-ph/0007346; R. Barbieri, hep-ph/9901228; D. Black et al., hep-ph/0004105; E. Akhmedov, Phys. Lett. B478, 215 (2000); M. Jezabek and Y. Sumino, Phys. Lett. B457, 139 (1999 ); H. Fritzsch and Z. Xing, hep-ph/9912358; and references therein.

[12] Incomplete list of references; K. S. Babu and S. M. Barr, Phys. Rev. Lett. 85, 1170 (2000); R. Barbieri et al., Nucl. Phys. B559, 17 (1999); A. Ghosal, hep-ph/9905470; D. Falcone, Phys. Lett. B475, 92 (2000); L. Lavoura, hep-ph/0005321; S. Lola and J. D. Vergados, hep-ph/9808269; S. K. Kang and C. S. Kim, Phys. Rev. D59, 091302 (1999); J. K. Elwood, et al., Phys. Rev. Lett. 81, 5064 (1998); K. Hagiwara and N. Okamura, Nucl. Phys. B548, 60 (1999); G. Altarelli and F. Feruglio, Phys. Lett. B451, 388 (1999); C. H. Albright, et al., Phys. Rev. Lett. 81, 1167 (1998); J. Ellis, et al., Eur. Phys. J. C 9, 389 (1999); M. Fukugita et al., Phys. Rev. D59, 113016 (1999); M. Abud et al., hep-ph/9911238; K. Choi et al., Phys. Rev. D60, 031301 (1999); S. M. Barr and I. Dorsner, hep-ph/0003058; M. Tanimoto, hep-ph/0008095; hep-ph/9910261; Z. Berezhiani and A. Rossi, hep-ph/0003084.

[13] L. Baudis et al., Phys. Rev. Lett. 83, 41 (1999).

[14] H. V. Klapdor-Kleingrothaus, J. Hellmig and M. Hirsch, J. Phys. G 24, 483 (1998).

[15] H. V. Klapdor-Kleingrothaus, hep-ex/9907040; L. Baudis et al., [GENIUS Collaboration], hep-ph/9910205.

[16] H. Georgi and S. L. Glashow, Phys. Rev. D61, 097301 (2000).

[17] S. K. Kang, C. S. Kim and J. D. Kim, Phys. Rev. D62, 073011 (2000).

[18] V. Barger, S. Pakvasa, T. J. Weiler and K. Whisnant, Phys. Lett. B437, 107 (1998).

[19] M. Bando, T. Kugo and K. Yoshioka, Phys. Rev. Lett. 80, 3004 (1998); S. M. Barr and I. Dorsner, hep-ph/0003058.

[20] see also D. Ng and Y. Ng, Mod. Phys. Lett. A6, 2243 (1991); G. Giudice, Mod. Phys. 
Lett. A7, 24291992; W. Hou and G. Wong, Phys. Rev. D52, 5269 (1995).

[21] A. Brignole, H. Murayama, and R. Rattazzi, Phys. Lett. B335, 345 (1994); F. Vissani and A. Yu. Smirnov, Phys. Lett. B341, 173 (1994).

[22] R. N. Mohapatra et al., Phys. Lett. B474, 355 (2000).

[23] M. Sakuda [K2K collaboration], in Proceedings of the APCTP workshop on Pacific Particle Physics Phenomenology, Seoul National University, Seoul, Korea, 1997.

[24] G. L. Fogli et al., Phys. Rev. D59, 033001 (1999). 